\title{
Hereditary Megaduodenum Presenting as Superior Mesenteric Artery Syndrome
}

Lucy Jamieson*, Sarah Jones

*Dalhousie Medical School Class of 2005

Abstract: A 15-year-old boy presenting with duodenal dilatation consistent with superior mesenteric artery syndrome (SMAS) is described. He was successfully treated with duodenojejunostomy but the operative findings were consistent with hereditary megaduodenum. Complications included esophagitis and gastroesophageal reflux that led to Barrett's esophagus. The unique presentations of hereditary megaduodenum and SMAS will be discussed.

A 15 -year-old boy presented with a 10-year history of intermittent vomiting and weight loss. The symptoms had significantly increased over the last few months and prior to presentation; the patient had postprandial abdominal pain relieved with vomiting. The patient had normal bowel function. He had a weight loss of 10 to $15 \mathrm{lbs}$ over the four previous months. The patient's past medical history and physical exam were unremarkable.

An upper GI series and follow-through showed a dilated first and second portion of the duodenum with a sharply demarcated cut-off zone consistent with SMAS. ${ }^{3}$ The following day a laparotomy and duodenojejunostomy were performed. Post-operatively, the patient's obstructive symptoms continued and he was treated with prokinetic agents to no effect. The patient was taken to the operating room two weeks later for a repeat gastroscopy and laparotomy. The esophagus was noted to have evidence of active esophagitis. At laparotomy, the duodenum was dilated beyond the superior mesenteric artery, a finding that was inconsistent with SMAS. At that time a duodenojejunostomy to the left of the mesenteric vessels was created. A third laparotomy and duodenojejunostomy were performed, proximal to the first. An appendectomy was also performed. Ten days later the anastomoses began to open up and the patient was able to tolerate a normal diet. Post-operatively the patient underwent a number of upper GI endoscopies that showed grade 3 esophagitis and severe gastroesophageal reflux. The patient was successfully managed with ranitidine. Complete healing of the esophagitis occurred; however, there was a ring of proximal migration of gastric epithelium along the site of previously inflamed esophagus suggestive of Barrett's epithelium. Biopsies confirmed Barrett's esophagus with no euplastic changes.

\section{Discussion}

Superior mesenteric artery syndrome is a condition in which the third portion of the duodenum is compressed by the overlying superior mesenteric artery, resulting in obstruction and dilatation. Factors thought to be involved in the pathogenesis include loss of fatty tissue in the mesentery around the artery as it crosses the duodenum, an increased angle between the artery and the aorta, and an upward displacement of the duodenum from an abnormally high ligament of Tritz. ${ }^{1}$ Predisposing factors include weight loss or rapid growth, spinal surgery, cerebral injury and burns. ${ }^{1}$ Symptoms are variable but may include upper abdominal pain, anorexia, early satiety, and postprandial vomiting. ${ }^{1,2}$

In some cases, SMAS can mimic other causes of duodenal obstruction such as megaduodenum. ${ }^{3}$ Hereditary megaduodenum is a rare autosomal dominant disease characterized by visceral myopathy of the digestive and urinary tracts presenting as pseudo-obstruction. Clinical presentation occurs equally in males and females with a mean age at diagnosis of 38 years. ${ }^{3}$ Symptoms of nausea, vomiting, abdominal pain and distention are intermittent and worsen following a meal. Bowel changes may include diarrhea, constipation, and steatorrhea. Some of these symptoms may be attributed to colonization of the hypotonic proximal small bowel. ${ }^{3}$ Patients may also be asymptomatic.

Histological studies of hereditary megaduodenum reveal no characteristic lesion. Ganglion cells are usually present. Interstitial fibrosis of the muscular layer with vacuolation of the muscle cells may either be responsible for the dysmotility or secondary to an underlying neural or metabolic disorder. Plain radiographs may suggest mechanical obstruction while barium studies suggest abnormal motility of most of the gastrointestinal tract. A high rate of spontaneous chromosomal damage in blood lymphocytes of patients with hereditary megaduodenum suggests an increased cancer risk. ${ }^{3}$

Medical management of hereditary megaduodenum includes treatment of symptoms, dietary management including a low-fiber, high-calorie diet, broad-spectrum antibiotics to control bacterial overgrowth, and nasogastric suction in acute cases. Surgery may include a duodenojejunostomy or a partial duodenal resection. Recently, Endo et al. used a surgical technique involving subtotal duodenectomy with a jejunal patch for two children with megaduodenum. ${ }^{4}$ The diseased duodenal wall was resected and reconstructed with the jejunum as an onlay patch. The patients showed markedly improved quality of life and catch-up growth after the operations. The authors concluded that this technique may be useful in functional duodenal obstruction with megaduodenum secondary to congenital duodenal malformations leading to blind loop syndrome .

The differential diagnosis of megaduodenum includes obstructive diseases with similar histological and biochemical lesions such as Hirschsprung's disease, Chaga's disease, scleroderma, intestinal amyloidosis, and a number of idiopathic diseases such as chronic idiopathic intestinal pseu- 
doobstruction (CIIP). Other causes of intestinal obstruction include atresias, congenital bands, stenoses, foreign bodies, adhesions and tumours. The differential can also include peptic ulcers, achalasia, various malabsorption syndromes and endocrinopathies.

Our patient presented with duodenal dilatation consistent with SMAS. He was treated with a duodenojejunostomy but the operative findings showed dilatation beyond the superior mesenteric artery, suggesting hereditary megaduodenum. It is most likely that our patient had a 10 year history of symptoms due to hereditary megaduodenum, followed by a recent significant history of weight loss that predisposed him to the development of SMAS. Complications of the chronic duodenal obstruction included grade 3 esophagitis and severe gastroesophageal reflux that progressed to Barrett's esophagus. This case study supports the need for clarification of the causes of intestinal obstruction in children, as delay in the diagnosis of SMAS as well as megaduodenum can result in malnutrition, dehydration and electrolyte abnormalities. ${ }^{1,3}$

\section{References}

1. Veysi VT, Humphrey G, Stringer MD. Superior mesenteric artery syndrome presenting with acute massive gastric dilation. J of Ped Surg 1997; 32(12): 1801-1803.

2. Delgadillo X, Belpaire-Dethiou M, Chantrain C et al. Arteriomesenteric syndrome as a cause of duodenal obstruction in children with cerebral palsy. J of Ped Surg 1997; 32(12): 1721-1723.

3. Basilisco G. Hereditary megaduodenum. Am J Gastroenterol 1997; 92(1):150-153.

4. Endo M, Ukiyama E, Yokoyama J, et al. Subtotal duodenectomy with jejunal patch for megaduodenum secondary to congenital duodenal malformation. J of Ped Surg 1998; 33(11): 1636-1640.

5. Shaw A, Shaffer H, Teja K, et al. A perspective for pediatric surgeons: Chronic idiopathic intestinal pseudoobstruction. J of Ped Surg 1979; 14(6): 719-725.
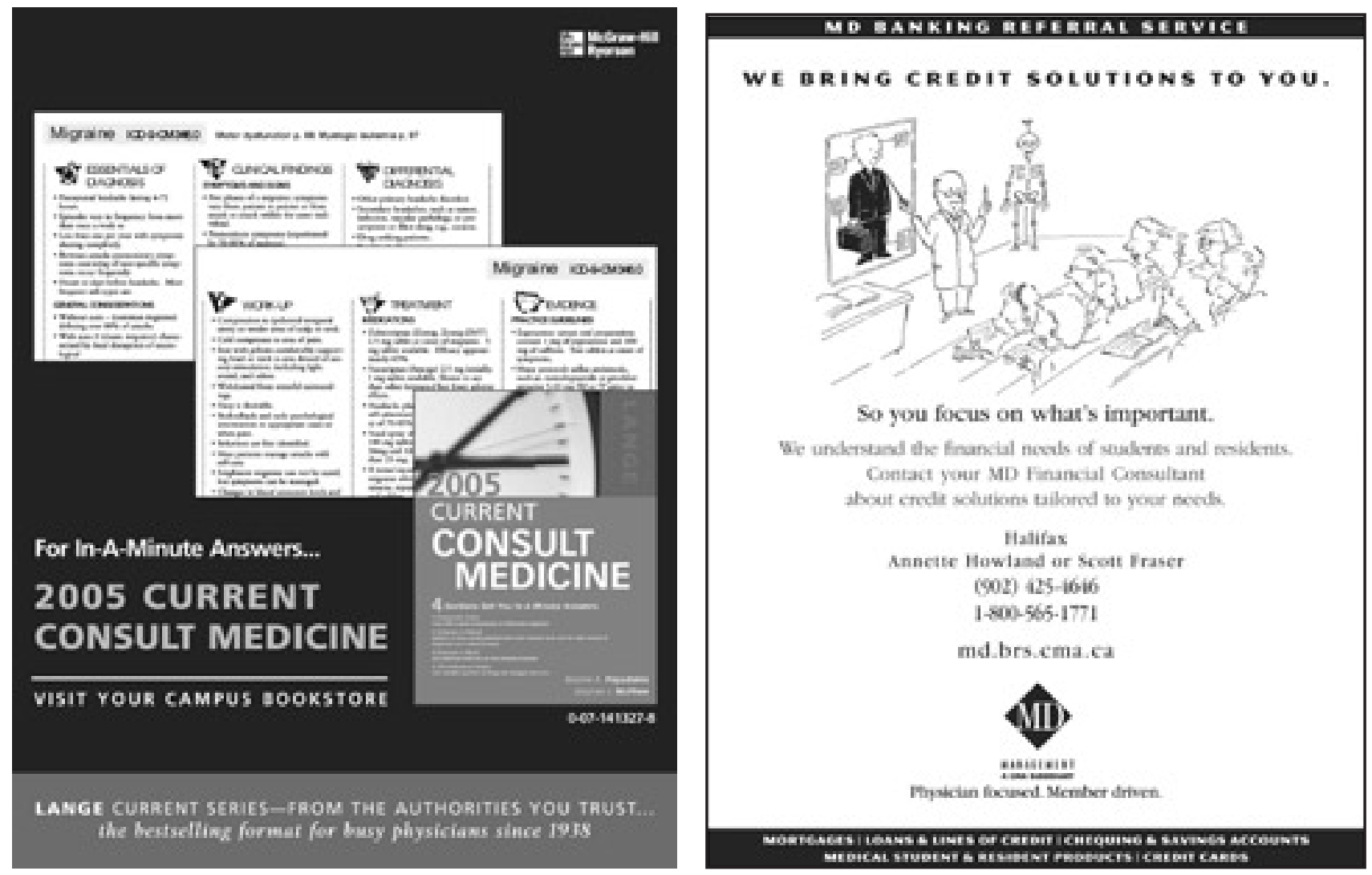